\title{
Measuring visfatin levels in saliva: an alternative approach to gestational diabetes screening
}

Hacer EROGLU IÇLi'

https://orcid. org/0000-0002-9775-1710

Tevfik Berk BíLDACl ${ }^{2}$

https:///orcid.org/0000-0002-6432-6777

\begin{abstract}
Objective: Oral glucose tolerance testing (OGTT) is the current recommended approach for the diagnosis of gestational diabetes mellitus (GDM). Visfatin is a type of novel adipokine of interest that mostly participates in glucose metabolism and inflammatory processes. We aim to identify a screening technique for GDM using salivary visfatin levels and to establish this technique's value as a screening method compared to OGTT. Mlaterials and methods: This is a cross-sectional case-control study. The cohort was formed from the saliva samples of pregnant patients in their $24^{\text {th }}$ through $28^{\text {th }}$ weeks of gestation. Patients were divided into two groups depending on their GDM status. OGTT and visfatin test results were compared and subjected to further analysis to establish a cutoff value for visfatin testing. Results: ELISA results indicated a significant difference between patients with GDM compared to patients without GDM; the values were $18.89 \pm 9.59$ and $12.44 \pm 8.75$, respectively (p: 0.007 ). A cutoff value of $10.5 \mathrm{ng} / \mathrm{mL}$ can be used to detect GDM with $78 \%$ sensitivity and $51 \%$ specificity. Conclusion: Salivary visfatin levels were significantly higher in patients with GDM. The existence of a differential in the concentration of visfatin in saliva can be utilized to develop a new screening method for GDM. Arch Endocrinol Metab. 2021;65(6):747-51
\end{abstract}

Keywords

Adipokine; gestational diabetes; oral glucose tolerance testing; visfatin
${ }^{1}$ Republic of Turkey Ministry of Health, Sultan Abdulhamid Han Education and Research Hospital, Division of Biochemistry, Istanbul, Turkey ${ }^{2}$ Baskent University Obstetrics and Gynecology Department, Istanbul Education and Research Hospital, Istanbul, Turkey
Correspondence to: Tevfik Berk BILDACl Baskent University Hospital Obstetrics and Gynecology Department Altunizade

Oymacı Sokak No. 7, Üsküdar, Istanbul, Turkey berkbildaci@gmail.com

Received on May/5/2020 Accepted on Jun/18/2021

\section{INTRODUCTION}

$\mathrm{D}$ uring pregnancy, patients are prone to many complications that can emerge during this period, such as gestational diabetes mellitus (GDM). GDM is a common complication defined as glucose intolerance initiated during pregnancy with no previous history of glucose intolerance. Following the adoption of International Association of Diabetes and Pregnancy Study Group's recommendations, the prevalence of GDM in developed countries was found to be around $18 \%$, an increase of about $40 \%$ from previous estimates between 1989 and 2004 (1,2). Oral glucose tolerance testing (OGTT) is an accepted method of testing and identifying glucose intolerance in pregnant patients that is recommended by various organizations such as the American College of Obstetrics and Gynecology (ACOG) and the American Diabetes Association (ADA) $(3,4)$.

Adipokines are peptides and proteins with various functions. They generally perform various tasks related to vascular hemostasis and blood pressure regulation (5).
Adipose tissue is an active endocrine organ that plays a substantial role in the specific hormone production of adipokines such as visfatin, which are believed to participate in energy storage. Dysregulation of pathways involving visfatin peptides has been observed to cause alterations in insulin sensitivity (6).

Visfatin can be encountered throughout the literature under various alternative names such as Pre-B-cell colony-enhancing factor l (PBEFl) and nicotinamide phosphoribosyl transferase (NAMPT). PBEFl is a protein originating from visceral adipose tissue that exists in both humans and mice, and which is a contributing factor to the rate-limiting step in the nicotinamide adenine dinucleotide $(\mathrm{NAD}+)$ salvage pathway. This pathway plays a role in converting nicotinamide to nicotinamide mononucleotide to enable $\mathrm{NAD}+$ biosynthesis $(7,8)$. Expression of visfatin is also observed in placental tissue, but in significantly lower quantities compared to its expression in subcutaneous and visceral adipose tissue. Visfatin secretion is strongly influenced by blood glucose levels in humans. Obesity 
is another factor that is associated with increased visfatin levels through an insulin-like effect in which it binds to the insulin receptor- 1 and where hypoglycemia is acquired through mechanisms involving the reduction of glycogenolysis and stimulation of glucose utilization $(9,10)$. Circulating visfatin levels are increased in patients with type 2 diabetes, metabolic syndrome, and/or cardiovascular diseases (11).

Adipokines can also be detected in saliva. A limited number of studies show a positive correlation between salivary levels of visfatin and other adipokines such as ghrelin and resistin in patients with type 2 diabetes mellitus (12). It was also recently established that levels of adipokines, including visfatin, are positively correlated with inflammatory conditions involving periodontal tissue, such as chronic periodontitis $(13,14)$. In this study, our goal is to identify whether a relationship exist between salivary visfatin levels and OGTT results showing GDM or a lack thereof.

\section{MATERIALS AND METHODS}

This is cross-sectional, case-control study was performed at Baskent University Istanbul Education and Research Hospital. The cohort was formed from saliva samples of patients in between their $24^{\text {th }}$ through $28^{\text {th }}$ weeks of pregnancy. Samples were collected using specialized collection tubes (Salivette ${ }^{\circledast}$, Sarstedt AG \& Co., Germany) (Figure 1) prior to initiating the patients' OGTT. Patients who were present for their routine prenatal visit for GDM screening in a low-risk clinical setting were asked to participate in this study during the allocated period. Collection time varied between 9 AM and 11 AM. Prior to saliva collection, patients were examined for signs of gingivitis by a dental practitioner with $5+$ years of experience in field. Patients found to have gingivitis were excluded from the study. Saliva samples were collected during a 3 -minute time frame using the collection tubes cited earlier. All saliva samples were centrifuged at $1000 \mathrm{G}$ for 20 minutes at temperatures between $2{ }^{\circ} \mathrm{C}$ and $8{ }^{\circ} \mathrm{C}$. This saliva supernatant was divided into $1-\mathrm{mL}$ aliquots in cryotubes. Specimens were immediately frozen to a temperature of $-80{ }^{\circ} \mathrm{C}$ and kept under same conditions until adipocytokine analysis. Immediately before the analysis, samples were thawed to room temperature, or $22{ }^{\circ} \mathrm{C}$.

The presence of GDM was assessed using $75-\mathrm{g}$ oral glucose solution and diagnosed when any one

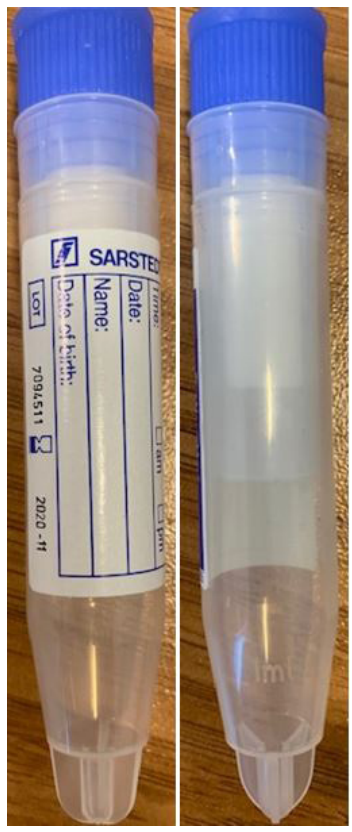

Figure 1. Images of saliva collection tubes.

of the following three results were identified: fasting $\geq 92 \mathrm{mg} / \mathrm{dL}(5.1 \mathrm{mmol} / \mathrm{L})$, l-hour glucose level $\geq 180 \mathrm{mg} / \mathrm{dL}(10.0 \mathrm{mmol} / \mathrm{L})$, or 2 -hour glucose level $\geq 153 \mathrm{mg} / \mathrm{dL}(8.5 \mathrm{mmol} / \mathrm{L})$. Patients diagnosed with GDM were initially treated with nutritional recommendations by a dietitian, if appropriate. If nutritional therapy was found to be ineffective or inadequate for achieving the target glucose levels (i.e., fasting $\leq 95 \mathrm{mg} / \mathrm{dL}, 1$ hour $\leq 140 \mathrm{mg} / \mathrm{dL}$, and 2 hours $\leq 120 \mathrm{mg} / \mathrm{dL}$ ), insulin therapy was initiated in consultation with an endocrinology specialist.

Visfatin levels were tested using an enzymelinked immunosorbent assay (ELISA) kit (Bioassay Technology Laboratory/Cat no. E0025, Hu Shanghai Korain Biotech Co., Ltd., Shanghai 200090, China). Inter- and intra-assay coefficients of variation (CV) were calculated to be $<10 \%$ and $<10 \%$, respectively. We preferred studying saliva samples rather than serum because of the novelty of salivary assessment and patients' desire for an easily applicable, non-invasive testing choice on GDM diagnosis.

In total, 91 patients provided appropriate consent for their samples' use in the study. This study was deemed in compliance and approved by the Baskent University Ethical Committee as KA19/395. SPSS version 23.0 was used for statistical analysis (IBM Corp. Released 2015. IBM SPSS Statistics for Windows, Version 23.0. Armonk, NY: IBM Corp.). An independent Student's t-test, Pearson correlation analyses, a ROC curve, and an AUC analysis were used where appropriate. 


\section{RESULTS}

Patients diagnosed with GDM were assigned to the study group (SG), which included 18 patients, whereas patients with normal OGTT values were assigned to the control group (CG), which included 73 patients. We lost touch with 17 patients during pregnancy follow-ups, so their childbirth outcomes were not included in the analysis. A post hoc analysis was performed following saliva sample collection, and a power of 0.92 with an effect size of 0.8 was achieved with the two groups' sample sizes. The incidence of GDM was $19.8 \%$. The mean age of the SG was 34.84, and the mean age of the CG was 32.05 (p: 0.035). Mean of patient properties and birth related outcomes were outlined in Table 1.

The ELISA results showed a significant difference between the two groups; SG: $18.89 \pm 9.59 \mathrm{ng} / \mathrm{mL}$ and CG: $12.44 \pm 8.75 \mathrm{ng} / \mathrm{mL}$ (p: 0.007). When age was tallied as an effecting cofactor, there was still a significant difference between two groups, as p: 0.009 in a controlled analysis. When OGTT results are analyzed individually for every hour and for fasting values, among the three values tested during the OGTT, only the 1-hour blood glucose level correlated significantly with visfatin levels. The results of the correlation analyses are listed in Table 2.

ROC (receiver operating characteristics) curve and AUC (area under curve) analyses were performed to identify a reasonable cutoff value for visfatin levels. AUC was observed to be 0.705 , with significance level of p: 0.007 and $95 \%$ CI (0.574 and 0.836 - lower and upper bounds, respectively). Using the point closest to the upper left corner of the chart, where sensitivity and specificity are greatest at their own values, $13.5 \mathrm{ng} / \mathrm{mL}$ was set as a cutoff value, achieving a sensitivity of $72 \%$ and a specificity of $63 \%$. Other sample values for the cited specifications are shared in Figure 2.

\section{DISCUSSION}

The relatively high prevalence of GDM, which ranges from $18 \%$ to $30 \%$ and increases in likelihood with a patient's age, makes this issue a priority, especially considering its associated negative economic impact and potential severe pregnancy complications (15). We observed an incidence of $19.8 \%$ among our patients, with a significant age difference between groups similar to that observed in a previous study of ours (16).

Even though treating GDM decreases the risk of associated complications, making the diagnosis as early as possible in pregnancy is of extreme importance. To overcome the heterogeneity in the diagnosis, universal OGTT based screening is employed to identify pregnant women with GDM. In general, adipokines, which play significant roles in glucose metabolism, can be utilized in the diagnosis of patients with GDM. Although their secretion might be altered by obesity, potentially making adipokine studies unreliable, numerous other studies have identified significant variations in adipokine levels in patients with GDM (17-19). Because studies

Table 1. Mean comparisons of patients' study-relevant properties and childbirth data

\begin{tabular}{lccc} 
& $\begin{array}{c}\text { Study Group (SG) } \\
\text { n: 18 }\end{array}$ & $\begin{array}{c}\text { Control Group (CG) } \\
\text { n: 73 }\end{array}$ & Significance (p) \\
\hline Age & $34.83 \pm 4.95$ & $32.05 \pm 4.93$ & .035 \\
Body mass index (BMI) & $28.18 \pm 2.68$ & $27.06 \pm 2.77$ & .125 \\
Gestational weeks at study & $25.47 \pm 1.14$ & $25.08 \pm 1.38$ & .269 \\
Birth week (CG missing 17 patients) & $38.23 \pm 1.47$ & $38.62 \pm 1.12$ & .233 \\
Birth weight in grams (CG missing 17 patients) & $3297 \pm 521$ & $3244 \pm 365$ & .633 \\
\hline
\end{tabular}

* Significant values are indicated in bold.

Table 2. Independent correlation of visfatin values with OGTT values

\begin{tabular}{lcccc}
\hline Correlations & Visfatin & Fasting Glucose & Glucose First Hour & Glucose Second Hour \\
\hline Visfatin & 1 & .102 & .214 & .183 \\
Fasting Glucose & .137 & 1 & $\mathbf{. 5 2 0}$ & $\mathbf{3 9 1}$ \\
Glucose First Hour & .211 & .520 & 1 & .762 \\
Glucose Second Hour & .180 &. $\mathbf{3 9 1}$ & $\mathbf{. 7 6 2}$ & 1 \\
\hline
\end{tabular}

* Significant values are indicated in bold (significance level $<0.05$ ). 


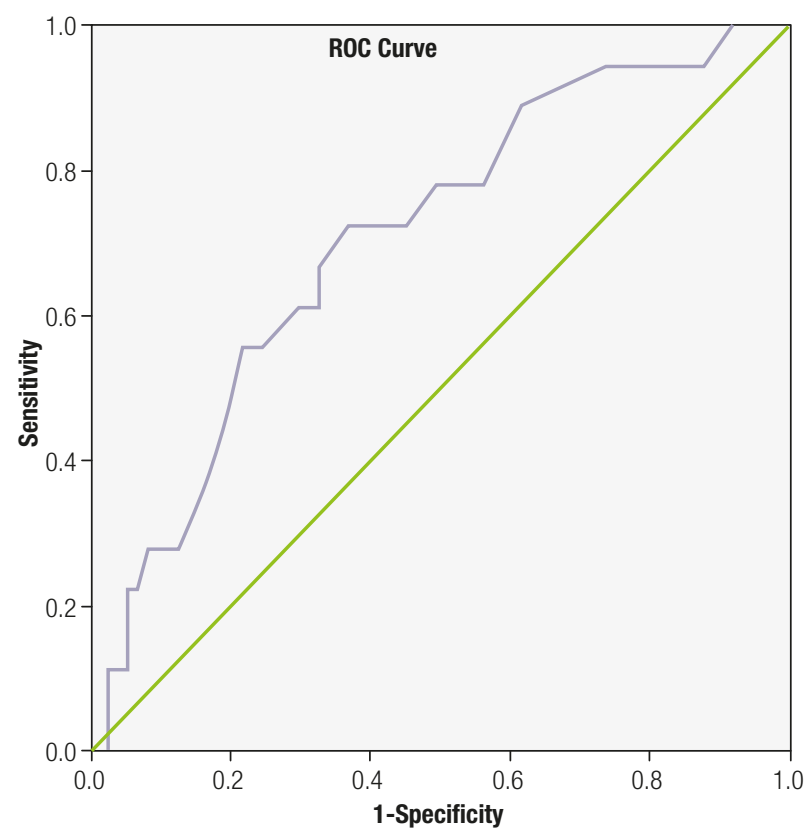

Area under curve (AUC) value: 0.705 (p: 0.007$)$ (Cl: 95\%

\begin{tabular}{ccc}
\hline Cutoff Values & Sensitivity & 1-Specificity \\
\hline $2.5 \mathrm{ng} / \mathrm{mL}$ & 1.0 & 0.92 \\
$6.5 \mathrm{ng} / \mathrm{mL}$ & 0.94 & 0.74 \\
$10.5 \mathrm{ng} / \mathrm{mL}$ & 0.78 & 0.49 \\
$13.5 \mathrm{ng} / \mathrm{mL}$ & 0.72 & 0.37 \\
$18.5 \mathrm{ng} / \mathrm{mL}$ & 0.56 & 0.22 \\
$31.5 \mathrm{ng} / \mathrm{mL}$ & 0.11 & 0.027 \\
\hline
\end{tabular}

Figure 2. ROC Curve and AUC analyses for visfatin values used to identify GDM.

involving adipokines were mostly performed using serum samples, our study employs a novel approach by using saliva samples to diagnose GDM.

The role of visfatin in the human body is not clearly established. However, these adipokines are involved in the regulation of energy homeostasis, which is the pathogenesis of diabetes mellitus. A strong metaanalysis by Chang and cols. showed that plasma visfatin concentrations were elevated in patients diagnosed with type 2 diabetes mellitus, metabolic syndrome, and cardiovascular diseases (20). In addition, Mopidevi and cols. observed that visfatin levels decreased following periodontitis treatment (13). Because the inclusion of patients with gingivitis would create a bias, we chose to exclude patients diagnosed with gingivitis. As the samples were collected from saliva, the matter of periodontitis was of utmost importance for us.
In a case-control study by Karatas and cols. that examined gestational weeks similar to those in our study, visfatin values detected in serum were not significantly different between patients with and without GDM (21). Because visfatin is secreted by epithelial cells of the amniotic sac during pregnancy, another group of researchers tested visfatin serum levels in patients in their 12th through 15 th weeks of pregnancy and was unable to identify a significant difference between patients who later developed GDM and those who did not $(22,23)$. However, Rezvan and cols. found significantly lower serum levels of visfatin in patients using OGTT (24). Therefore, we can assume that data about serum levels for visfatin and their use in establishing glucose intolerance remain controversial in the literature. This is one of the reasons we conducted this study on a novel technique using saliva samples.

A recent study of patients in early pregnancy performed by Bawah and cols. observed that visfatin, along with other adipokines such as resistin and leptin, is significantly more useful in predicting patients who will eventually be diagnosed with GDM in their 24th through 28 th weeks. The same study also revealed the data for visfatin levels when predicting GDM in the 24 th through 28 th weeks with a sensitivity of $87.1 \%$ and a specificity of $70 \%(5)$. Our results for sensitivity and specificity are close, but they are less than what they observed. However, we think lower values can be associated with decreased passage from serum to saliva or the gestational week differences during the visfatin collection period.

In conclusion, in the search for a simple, costeffective, non-invasive, patient-friendly technique, saliva must be considered as a specimen because it is easy to collect, simple to use, and requires no invasive collection procedures. To the best of our knowledge, this study is the first to use visfatin adipokine to diagnose GDM using saliva samples. In our study, we believe that observing a significant difference in visfatin values among patients with GDM was a big step. However, we were unable to establish a sensitivity and specificity rate high enough to replace OGTT for GDM testing. Even so, with further studies on the horizon that incorporate analyzing larger samples of the population and other molecules, especially other adipokines, physicians' preferences might shift toward saliva testing instead of OGTT.

Acknowledgements: we, the authors, would like to thank the hardworking staff of Baskent University's laboratory, especially their supervisors Nazan Camca Sen and Ali Inal, M.D., for their 
dedicated work and the time they shared while this manuscript was being drafted. We would also like to thank Guldeniz Desteli, M.D., and Berkem Okten, M.D., for inviting their patients to participate in the study, which accelerated the cohort formation process.

Funding: this study was self-funded by the authors.

Disclosure: no potential conflict of interest relevant to this article was reported.

\section{REFERENCES}

1. Getahun D, Nath C, Ananth CV, Chavez MR, Smulian JC. Gestational diabetes in the United States: temporal trends 1989 through 2004. Am J Obstet Gynecol. 2008;198(5):525.e1-5.

2. Cheung NW, Jiang S, Athayde N. Impact of the IADPSG criteria for gestational diabetes, and of obesity, on pregnancy outcomes. Aust N Z J Obstet Gynaecol. 2018;58(5):553-9.

3. American Family Physician. Practice Guidelines - ACOG Releases Guideline on Gestational Diabetes. Am Fam Physician. 2014;90(6):416-7.

4. American Diabetes Association. Standarts of Medical Care in Diabetes - 2016. Diabetes Care. 2016;39(Suppl 1):1-106.

5. Bawah AT, Seini MM, Abaka-Yawason A, Alidu H, Nanga S. Leptin, resistin and visfatin as useful predictors of gestational diabetes mellitus. Lipids Health Dis. 2019;18(1):221.

6. YamauchiT, Kamon J, Waki H, TerauchiY, Kubota N, Hara K, et al.The fatderived hormone adiponectin reverses insulin resistance associated with both lipoatrophy and obesity. Nat Med. 2001;7(8):941-6.

7. Revollo JR, Grimm AA, Imai S. The regulation of nicotinamide adenine dinucleotide biosynthesis by Nampt/PBEF/visfatin in mammals. Curr Opin Gastroenterol. 2007;23(2):164-70.

8. Ognjanovic S, Ku TL, Bryant-Greenwood GD. Pre-B-cell colony-enhancing factor is a secreted cytokine-like protein from the human amniotic epithelium. Am J Obstet Gynecol. 2005;193(1):273-82.

9. Adeghate E. Visfatin: structure, function and relation to diabetes mellitus and other dysfunctions. Curr Med Chem. 2008;15(18):1851-62.

10. Haider DG, Schaller G, Kapiotis S, Maier C, Luger A, Wolzt M. The release of the adipocytokine visfatin is regulated by glucose and insulin. Diabetologia. 2006;49(8):1909-14.

11. Radzicka S, Pietryga $M$, Iciek $R$, Brazert J. The role of visfatin in pathogenesis of gestational diabetes (GDM). Ginekol Pol. 2018;89(9):518-21.
12. Srinivasan M, Meadows ML, Maxwell L. Assessment of Salivary Adipokines Resistin, Visfatin, and Ghrelin as Type 2 Diabetes Mellitus Biomarkers. Biochem Res Int. 2018;2018:7463796.

13. Mopidevi A, Penmetsa GS, Dwarkanath CD, Dubba K, Gadde P. Salivary visfatin concentrations in patients with chronic periodontitis: An analysis before and after periodontal therapy. Indian J Dent Res. 2019;30(6):864-9.

14. Tabari ZA, Azadmehr A, Nohekhan A, Naddafpour N, Ghaedi FB. Salivary visfatin concentrations in patients with chronic periodontitis. J Periodontol. 2014;85(8):1081-5.

15. Shahbazian H, Nouhjah S, Shahbazian N, Jahanfar S, Latifi SM, Aleali A, et al. Gestational diabetes mellitus in an Iranian pregnant population using IADPSG criteria: Incidence, contributing factors and outcomes. Diabetes Metab Syndr. 2016;10(4):242-6.

16. Bildacı TB, Çevik H, Aksan Desteli G, Tavaslı B, Özdo囚an S. Placental elasticity on patients with gestational diabetes: Single institution experience. J Chin Med Assoc. 2017;80(11):717-20.

17. Fasshauer $M$, Bluher $M$, Stumvoll $M$. Adipokines in gestational diabetes. Lancet Diabetes Endocrinol. 2014;2(6):488-99.

18. Krzyzanowska K, Krugluger W, Mittermayer F, Rahman R, Haider D, Shnawa N, et al. Increased visfatin concentrations in women with gestational diabetes mellitus. Clin Sci (Lond). 2006;110(5):605-9.

19. Lowe LP, Metzger BE, Lowe WL Jr, Dyer AR, McDadeTW, McIntyre $H D$, et al. Inflammatory mediators and glucose in pregnancy: results from a subset of the Hyperglycemia and Adverse Pregnancy Outcome (HAPO) Study. J Clin Endocrinol Metab. 2010;95(12):5427-34.

20. Chang YH, Chang DM, Lin KC, Shin SJ, Lee YJ. Visfatin in overweight/obesity, type 2 diabetes mellitus, insulin resistance, metabolic syndrome and cardiovascular diseases: a meta-analysis and systemic review. Diabetes Metab Res Rev. $2011 ; 27(6): 515-27$.

21. Karatas A, Tuncay Isikkent N, Ozlu T, Demirin H. Relationship of maternal serum resistin and visfatin levels with gestational diabetes mellitus. Gynecol Endocrinol. 2014;30(5):355-8.

22. Abell SK, Shorakae S, Harrison CL, Hiam D, Moreno-Asso A, Stepto $\mathrm{NK}$, et al. The association between dysregulated adipocytokines in early pregnancy and development of gestational diabetes. Diabetes Metab Res Rev. 2017;33(8).

23. Ferreira AF, Rezende JC, Vaikousi E, Akolekar R, Nicolaides KH. Maternal serum visfatin at 11-13 weeks of gestation in gestational diabetes mellitus. Clin Chem. 2011;57(4):609-13.

24. Rezvan N, Hosseinzadeh-Attar MJ, Masoudkabir F, Moini A, Janani $\mathrm{L}$, Mazaherioun M. Serum visfatin concentrations in gestational diabetes mellitus and normal pregnancy. Arch Gynecol Obstet. 2012;285(5):1257-62. 